\title{
A genetic map of tetraploid Paspalum notatum Flügge (bahiagrass) based on single-dose molecular markers
}

\author{
Juliana Stein · Silvina C. Pessino · Eric J. Martínez • María Pía Rodriguez • \\ Lorena A. Siena · Camilo L. Quarin · Juan Pablo Amelio Ortiz
}

Received: 1 September 2006/ Accepted: 29 January 2007/Published online: 23 February 2007

(C) Springer Science+Business Media B.V. 2007

\begin{abstract}
Paspalum notatum Flügge is a warmseason forage grass with mainly diploid $(2 \mathrm{n}=$ $20)$ and autotetraploid $(2 n=40)$ representatives. Diploid races reproduce sexually and require crosspollination due to a self-incompatible mating system, while autotetraploids reproduce by aposporous apomixis. The objectives of this work were to develop a genetic linkage map of Paspalum notatum Flügge at the tetraploid level, identify the linkage/s group/s associated with apomixis and carry out a general characterization of its mode of inheritance. A pseudo test-cross $F_{1}$ family of 113 individuals segregating for the mode of reproduction was obtained by crossing a synthetic completely sexual tetraploid plant (Q4188) as female parent with a natural aposporous individual (Q4117) as pollen donor.
\end{abstract}

J. Stein · S. C. Pessino - M. P. Rodriguez .

L. A. Siena · J. P. A. Ortiz (ه)

Laboratorio de Biología Molecular, Facultad de Ciencias Agrarias, Universidad Nacional de Rosario

(UNR), CC 14, Zavalla, Pcia. de Santa Fe S2125ZAA, Argentina

e-mail: jortiz@unr.edu.ar

E. J. Martínez · C. L. Quarin · J. P. A. Ortiz Instituto de Botánica del Nordeste (IBONE)CONICET, Facultad de Ciencias Agrarias, Universidad Nacional del Nordeste (UNNE), CC 209, Corrientes 3400, Argentina
Map construction was based on single-dose markers (SDAFs) segregating from both parents. Two linkage maps (female and male) were constructed. Within each map, homologous groups were assembled by detecting repulsion-phase linked SDAFs. Putative Q4188 and Q4117 homolog groups were identified by mapping shared single dose markers (BSDF). The Q4188 map consisted of 263 markers distributed on 26 co-segregation groups over a total genetic distance of 1.590.6 cM, while the Q4117 map contained 216 loci dispersed on 39 co-segregation groups along 2.265.7 cM, giving an estimated genome coverage of $88 \%$ and $83 \%$, respectively. Seven and 12 putative homologous chromosomes were detected within Q4188 and Q4117 maps, respectively. Afterward, ten female and male homologous chromosomes were identified by mapping BSDFs. In the Q4117 map, a single linkage group was associated with apospory. It was characterized by restriction in recombination and preferential chromosome pairing. A BPSD marker mapping within this group allowed the detection of the female homolog and the putative four male groups of the set carrying apospory.

Keywords Apospory - Mapping in autopolyploids · Paspalum notatum - Single dose markers 


\section{Introduction}

Paspalum notatum Flügge (bahiagrass) is a perennial rhizomatous grass species naturally distributed from central eastern Mexico to Argentina and throughout the West Indies (Chase 1929). The species forms an agamic complex where diploid biotypes are sexual and self-incompatible while polyploids are self-fertile pseudogamous apomicts (Burton 1948). Sexual diploid races naturally inhabit a very limited area in South America (Daurelio et al. 2004). They were accidentally introduced into the United States, probably as a consequence of cattle transport (Burton 1967). This adventitious material was improved by conventional breeding and brought into cultivation as Pensacola bahiagrass (Burton 1967). Currently it became one of the major forage grasses grown in the Southern parts of the US (Espinoza et al. 2006). On the other hand, tetraploid races $(2 \mathrm{n}=4 \mathrm{x}=40)$ of $P$. notatum (common bahiagrass) are generally considered to be the typical form of the species and represent important natural forage resources for the tropical and subtropical areas of southern-Brazil, Paraguay and north-eastern Argentina (Gates et al. 2004). Although common bahiagrass has an excellent potential for forage production, genetic improvement has been difficult because of the asexual mode of reproduction and the lack of stable sexual tetraploids genotypes (Quarin et al. 2003). Cytogenetic observations and genetic analyses of AFLP markers segregation indicated that tetraploids have an autopolyploid origin and a general polysomic inheritance (Forbes and Burton 1961; Quarin et al. 1984; Stein et al. 2004).

Apomixis refers to a clonal form of propagation through seeds leading to the generation of progenies that are exact genetic replicas of the mother plant (Nogler 1984). Apomixis in $P$. notatum is characterized by the presence of aposporous embryo sacs derived from nucellar cells, the parthenogenetic development of the embryos and the formation of endosperm after the fertilization of the polar nuclei (pseudogamy) (Martínez et al. 2001). Genetic and molecular studies showed that in this species apospory is controlled by a single dominant locus with a distorted segregation ratio, which is located in a genomic region where recombination is restricted (Martínez et al. 2001; 2003). The apospory locus appeared to be included in a genetic block that presented preferential chromosome pairing, possibly indicating that it holds increased homology with only one of the other three homologous chromosomes of the set (Stein et al. 2004). Moreover, comparative analysis identified RFLP probes of rice chromosome 2 and 12 mapping strongly linked to apospory in $P$. notatum and revealed that the locus presents a partial conservation of synteny with other two species of the genus ( $P$. simplex and P. malacophyllum) (Pupilli et al. 2004). RFLP probes from rice chromosome 2 were also found linked to apospory in Brachiaria hybrids by Pessino et al. (1998).

A molecular markers-based genetic linkage map of sexual diploid bahiagrass (Pensacola bahiagrass $)(2 \mathrm{n}=2 \mathrm{x}=20)$ was already developed by our group (Ortiz et al. 2001). Map construction was based on a comparative approach that used an $F_{1}$ mapping family derived from noninbred parents and heterologous RFLP clones distributed over the maize and rice maps. RAPD and AFLP markers were included to consolidate the linkage groups. The integrated map contained the 10 expected linkage groups and extended along $991 \mathrm{cM}$ where several regions syntenic to maize and rice were identified (Ortiz et al. 2001). Since apomixis does not occur at the diploid level, we were unable to identify the genomic region associated to apospory.

Mapping in polyploids has been regarded as more complex than in diploids because of the higher number of possible genotypes, the difficulties associated with genotype identification and the poor definition of the polyploid constitution in many species (Ripol et al. 1999). Wu et al. (1992) designed a method based on the analysis of single dose restriction fragments (SDRF) to facilitate the mapping in such species. An SDRF (or alternatively a single dose amplification fragment SDAF) is defined as a DNA fragment present in just one chromosome that segregate in the progeny in a ratio of 1:1, regardless of the ploidy level or type. Using this strategy, mapping in autopolyploids usually is carried out in 2 steps: the ordering of loci along individual chromosomes and the assigning of the chromosomes to 
homologous groups (Ripol et al. 1999). Homologs can be identified either by mapping RFLP probes that recognize multiple single-dose fragments ( $\mathrm{Da}$ Silva et al. 1993), by using multiple-dose fragments (Ripol et al. 1999) or by detecting linkages in repulsion-phase between markers located on homologous chromosomes (Al Janabi et al. 1993). Genetic linkage maps of polyploid species based on single dose fragments have been developed for several species like Saccharum spontaneum (Da Silva et al. 1993), Poa pratensis (Porceddu et al. 2002), Fragaria $x$ ananassa (Lerceteau-Kohler et al. 2003), Pennisetum ciliare (Jessup et al. 2003), Festuca arundinacea (Saha et al. 2005), Panicum maximum (Ebina et al. 2005), Panicum virgatum (Missaoui et al. 2005) and Cynodon sp. (Bethel et al. 2006).

The objective of this work was to develop a genetic linkage map of tetraploid $P$. notatum with AFLP and RFLP markers to be used for fundamental genetic studies involving apomixis as well as in breeding programs of the cultivated tetraploid ecotypes.

\section{Materials and methods}

\section{Plant material}

The mapping population used was the same employed by Stein et al. (2004). It consisted of 113 hybrids derived from a cross between a completely sexual tetraploid genotype of experimental origin (Q4188) (Quarin et al. 2003) and a natural obligated aposporous plant (Q4117). Molecular and cytoembryological classification for the mode of reproduction of each $F_{1}$ progeny rendered 15 aposporous vs. 98 non-aposporous $\mathrm{F}_{1}$ plants (Stein et al. 2004).

\section{DNA markers}

DNA extractions were carried out using the CTAB method according to Martínez et al. (2003). AFLP markers were generated as described in Ortiz et al. (2001), by employing 32 primer combinations from the AFLP Protocol for Public Release, Key Gene, Version 2.0, Netherlands (1994). Polymorphic fragments were named ex/mx ( $\mathrm{x}=1$ to $\mathrm{n})$, designating the specific EcoRI and $M s e I$ primers used in the selective amplification, and a final letter to differentiate fragments derived from the same amplification. RFLP were generated as described by Ortiz et al. (2001) using anchored clones from maize (UMC-University of Missouri, Columbia, USA) and rice (RZAnchor set of University of Cornell, USA and C or R-New Landmarker set of the Rice Genome Research Program, Japan). Besides, three cDNA clones of P. notatum (ARP1, ARP2 and SRP1) (Pessino et al. 2001 and SC Pessino personal communication) and 7 from Brachiaria brizantha (No. 5, 7, 18, 20, 30, 34, 56) (Rodrigues et al. 2003) were tested as probes. All these fragments derived from mRNA transcripts differentially expressed during aposporous and non-aposporous embryo sac development. AFLP segregation data were taken from the complete progeny while RFLP ones from a sample of 80 individuals (all the aposporous and 65 randomly-chosen nonaposporous plants).

\section{Data analysis and linkage map construction}

Segregation data from each parental genotype were analysed independently. The presence/ absence of each polymorphic fragment was visually determined and recorded for each progeny. A $\chi^{2}$ test was used to determine the goodness of fit (at $P \leq 0.01$ ) between the observed and the expected number of genotypes for each class of segregation ratio. For single dose amplification fragments (SDAFs) that were polymorphic between parents (i.e. present in Q4188 and absent Q4117 or viceversa), the expected segregation ratio was $1: 1$, whatever the chromosome pairing configurations at meiosis (Wu et al. 1992). In the case of simplex markers homomorphic for parents but segregating in the population (biparental single dose fragments; BSDF), the expected ratio was 3:1 for either disomic or polysomic inheritance (Ritter et al.1990). The linkage map was based on the utilization of undistorted SDAFs $(P<0.01)$ derived from the pseudo-test cross mapping population (Grattapaglia and Sederoff 1994). Map units in centimorgan (cM) were derived from the Kosambi (1944) mapping function. Linkage analysis was performed with 
MAPMAKER/EXP 3.0 (Lander et al. 1987) at LOD threshold 6.0-2.0 and a recombination frequency $\theta=0.39$ [the maximum detectable recombination fraction (MaxR) for a population of 113 individuals from an autotetraploid ( $\mathrm{Wu}$ et al. 1992)]. Two framework genetic maps (female and male) were developed with SDAF linked in coupling phase (referred below as cosegregation groups) at LOD $=6.0-4.0$. The LOD value was then decreased to 3.0 to test for possible associations between co-segregation group ends. Association was accepted when one marker from a group was linked to more than one marker from the other group. Unlinked markers were added to the co-segregation groups by using the "assign" command. The "order" command was used to establish the maximum likelihood order of markers in each co-segregation and the "ripple" command to verify the order. Female and male groups were named with an $\mathrm{F}$ and $\mathrm{M}$ respectively, followed by a number and a letter for differentiating among linkage groups and homologs, respectively. Homologous chromosomes were detected by analysing repulsionphase linked markers according to Al-Janabi et al. (1993). Repulsion markers were generated by recoding the presence and absence data set of each SDAF and including the direct and inverted data sets together in the same input file for MAPMAKER/EXP 3.0. Linkages between a direct and inverted score (and viceversa) were considered as indicative of loci linked in repulsion-phase. Pairs of markers linked in repulsion belonging to different co-segregation groups were considered as being in homologous chromosomes. Finally, pair-wise linkage between SDAF (1:1) and BSDF (3:1) were examined using JOINMAP $1.4($ Stam 1993) at a minimum LOD $=3.0$. Data from BSDF markers were added to the SDAF data set and pair-wise linkage, 3:1 vs. 1:1, was detected individually. BSDF loci were named with an $\mathrm{X}$ followed by the corresponding AFLP code and added to the map as accessory markers in the more probable interval among SDAF given by JOINMAP 1.4. Mapped BSAF were used to determine female and male homologous co-segregation groups (Maliepaard et al. 1998). The ratio of pairs of markers linked in repulsion vs. coupling phase obtained by a two point analysis of the whole data set was used for estimating the type of inheritance (disomic/polysomic) in both parental genotypes. Ratios of 1:1 or $0-0.25-1$ were expected for disomic or polysomic inheritance respectively (Wu et al. 1992). Random or preferential chromosome pairing was estimated as described by Qu and Hancock (2001).

Estimation of genome size and coverage

The expected genome size $\mathrm{E}(\mathrm{G})$ was estimated by following the method proposed by Chakravarti et al. (1991), solving the equation: $E(G)=$ $[n(n-1) d] / k$, where $n$ is the number of markers on the map, $\mathrm{d}$ is the maximum distance between two markers at a given LOD score and $\mathrm{k}$ is the number of pairs of markers linked at the stated LOD score. Genome coverage $\mathrm{E}(\mathrm{C})$ was estimated as the ratio among the total length of the map obtained by adding all linkage groups vs. the expected genome size value $\times 100$.

\section{Results}

Segregation analysis of DNA markers

As a result of using a tetraploid $F_{1}$ mapping population derived from non-inbred parents, different allelic configurations per-locus were expected (Ritter et al. 1990). Segregation data were investigated by the presence of: (a) maternal markers (fragments segregating from Q4188), (b) paternal markers (fragments segregating from Q4117) or (c) biparental markers (fragments present in both parents and segregating in the progeny) (Table 1). The number of markers scored in each class of segregation ratio is shown in Table 2. In both genotypes, most polymorphic fragments segregated as SDAFs. Double-dose markers (DDAF) segregated mainly in a 5:1 ratio following a tetrasomic inheritance, but a group of them fit on a 3:1 ratio (disomic inheritance) (Table 2). Distorted markers were generated from both genotypes although they were more abundant in the aposporous parent (Table 2). Allelic configuration of the biparental makers detected is shown in Table 2. Sixty of them fitted with the segregation ratio expected for BSDF 
Table 1 Total AFLP and RFLP molecular markers scored on tetraploid Paspalum notatum Flügge mapping population

\begin{tabular}{llcl}
\hline Type of marker & AFLP & RFLP & Total \\
\hline Maternal & 307 & 9 & 316 \\
Paternal & 257 & 9 & 266 \\
Biparental & 103 & 2 & 105 \\
Total & 667 & 20 & 687 \\
\hline
\end{tabular}

(3:1). The histogram of frequencies of the complete data set of maternal and paternal markers scored is shown in Fig. 1. The distribution appeared to be bimodal, with a larger group in the area of 0.5 , the expected segregation ratio of SDAFs, and a smaller group around 0.78-0.83, which would correspond to the double-dose amplification fragments (DDAFs). In the region of 0.10-0.30 appeared those markers segregating from the aposporous parent that showed distorted ratios.

\section{Map of sexual Q4188}

The linkage map of Q4188 was based on 259 maternal SDAF loci (Table 2). Twenty seven linkage groups were first defined at LOD $=6.0$, but 26 definitive co-segregation groups of undistorted SDAF markers were formed when LOD

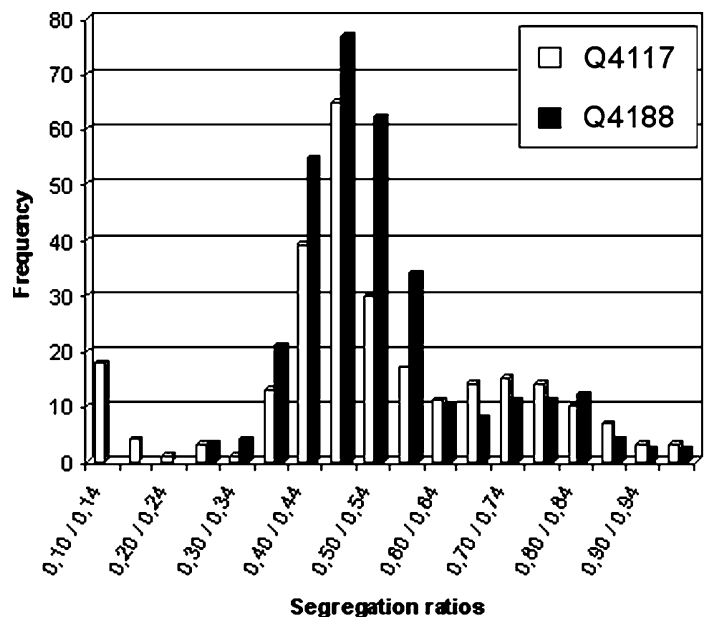

Fig. 1 Distribution of the observed segregation ratios for the 582 (female and male) markers scored in the tetraploid $P$. notatum mapping population. Segregation ratios are represented as the number of individuals with a specific marker/total number of individuals scored
Fig. 2 Linkage map of sexual tetraploid $(2 n=4 x=40)$ $P$. notatum genotype Q4188. Markers' names and distances in $\mathrm{cM}$ (Kosambi) are indicated on the right and the left, respectively. SDAFs co-segregation groups were mapped at LOD values 6.0-3.0. BSDF markers (underlined) were allocated at $\mathrm{LOD}=3.0$. Asterisks indicate markers showing distorted segregation ratios. Italics indicate markers added at $\mathrm{LOD}=2.0$ and brackets specify markers for which no unique place was found. Where possible, homologous groups were aligned on the basis of SDAF linked in repulsion-phase (horizontal lines). Letters indicate homologous chromosomes

was decreased to 3.0 (see Material and Methods). Remaining unlinked SDAFs were assigned at $\mathrm{LOD}=3.0$ and the more probable order of markers within each linkage group was determined. Distorted markers were added to the cosegregation groups at LOD $=3.0$. The framework maternal map consisted of 233 markers distributed in 26 co-segregation groups of two or more markers that spanned 1,590.6 cM (Fig. 2). The average genetic distance between markers was about $6.8 \mathrm{cM}$ and each group was defined by about 9 markers. From a total of 10 distorted markers, 6 mapped distributed in 4 different linkage groups and the remaining 4 grouped together on a single linkage group. Finally, two SDAFs were added at $\mathrm{LOD}=2.0$. Analysis of markers linked in repulsion-phase allowed the identification of 7 putative homologous chromosomes of 2 or 3 co-segregation groups. Besides, three previously unlinked SDAF markers showed linkage in repulsion-phase with markers corresponding to groups F1a, F9a and F11a. These single markers were incorporated to the map considering they belong to the putative homologous groups (Fig. 2). Distances detected for the majority of markers linked in repulsion-phase were higher than $33.3 \mathrm{cM}$ (the minimum value expected for tetrasomic inheritance) indicating that inheritance was mainly polysomic. Moreover, the ratio among the number of pairs of markers linked in repulsion vs. coupling phase (51/1277) corresponded to values expected for an autotetraploid (Qu and Hancock 2001). The estimation of the genome size at $\mathrm{LOD}=2.0$ resulted in $1,815 \mathrm{cM}$. This value indicated that the genome coverage by the recombination map was about of $87.6 \% \quad[(1,590.6 \mathrm{cM} / 1,815 \mathrm{cM}) \times 100]$. Thirty biparental markers were incorporated to 17 out 

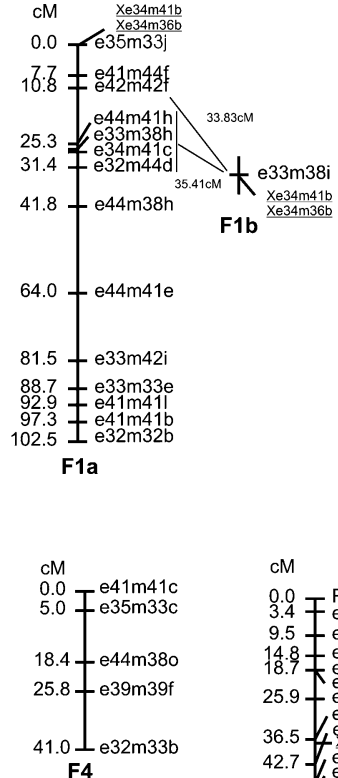

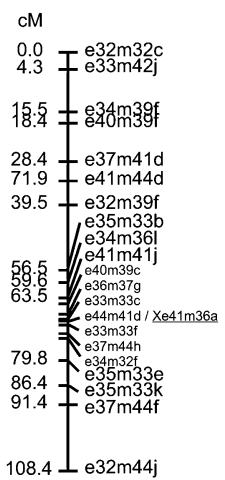

F2
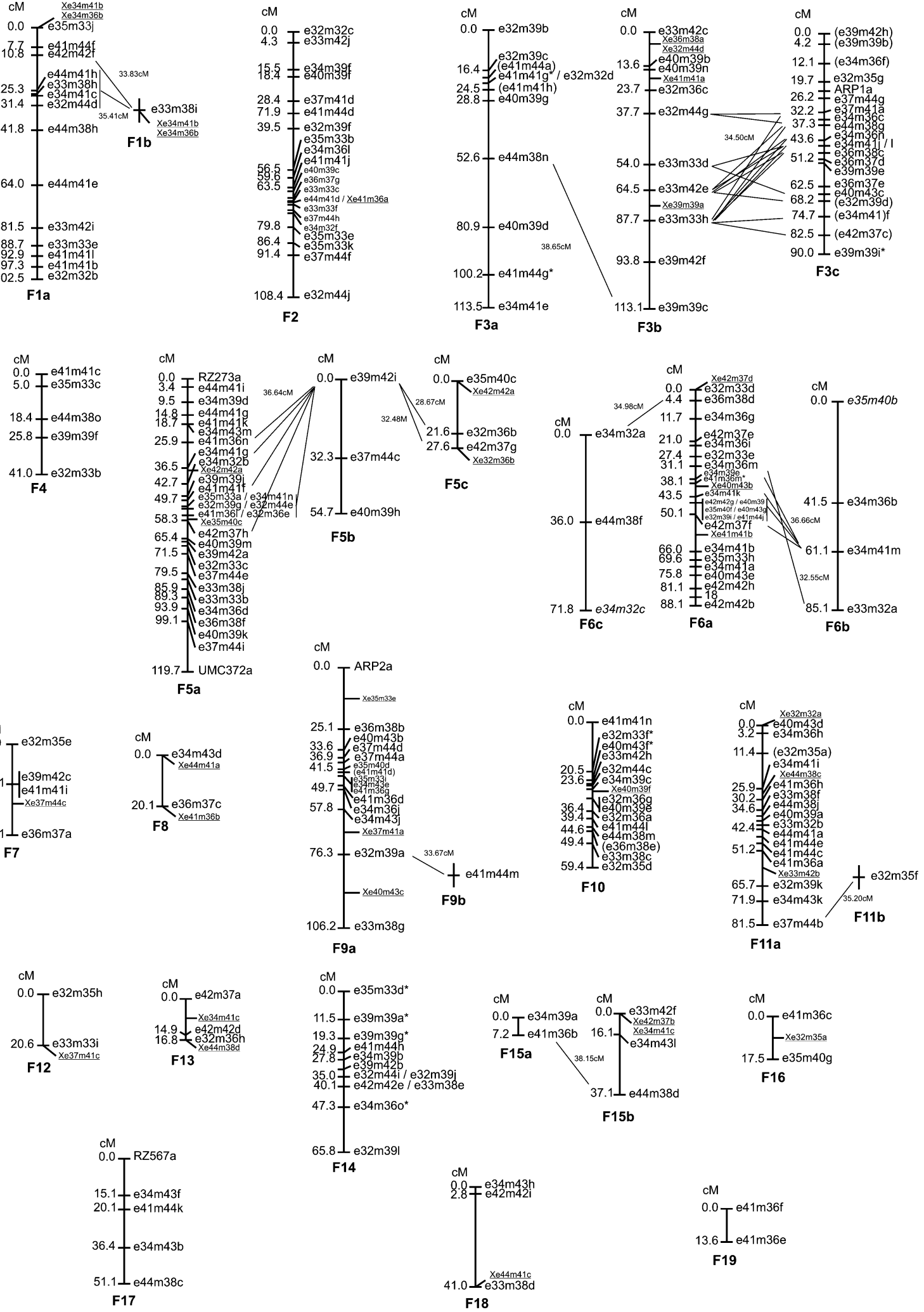
Table 2 Number of markers scored in each class of segregation ratio

\begin{tabular}{|c|c|c|c|c|c|}
\hline \multirow[t]{2}{*}{ Type of Marker } & \multicolumn{4}{|c|}{ Allelic configuration and expected segregation ratio } & \multirow[t]{2}{*}{ Total } \\
\hline & SDAF & DD & & Dist. $^{\mathrm{a}}$ & \\
\hline & $1: 1$ & $5: 1$ & $3: 1$ & & \\
\hline \multirow{4}{*}{$\begin{array}{l}\text { Maternal } \\
\text { Paternal }\end{array}$} & 259 & 29 & 12 & 16 & 316 \\
\hline & 177 & 39 & 15 & 35 & 266 \\
\hline & BSDF & \multicolumn{2}{|c|}{ BMDF } & \multicolumn{2}{|l|}{ Dist. $^{\mathrm{a}}$} \\
\hline & $3: 1$ & \multicolumn{2}{|c|}{$(11: 1 / 7: 1)+(35: 1 / 15: 1)^{b}$} & & \\
\hline Biparental & 60 & \multicolumn{2}{|l|}{39} & 6 & 105 \\
\hline
\end{tabular}

of the 26 previously defined groups by using JOINMAP 1.4 at LOD $=3.0$. These markers were included without indication of the genetic distance to avoid distortion in the 1:1 genetic frame and consequently were not considered for the estimation of the map length. Particularly, the linkage of three BSDF to markers placed in different co-segregation groups $(\mathrm{Xe} 34 \mathrm{~m} 41 \mathrm{~b}$ and $\mathrm{Xe} 34 \mathrm{~m} 36 \mathrm{~b}$ to markers in F1a and F1b; Xe42m42a to markers in F5a and F5c) confirmed the association of homologous groups previously detected by the repulsion-phase analysis (Fig. 2).

\section{Map of aposporous Q4117}

The map of Q4117 was based on the analysis of 177 paternal SDAF loci. Linkage analysis was carried out starting at LOD $=4.0$ where 42 initial co-segregation groups were identified. Association between co-segregation groups' ends resulted in the identification of 36 co-segregation groups of 2 or more markers. The remaining SDAFs were assigned at LOD $=3.0$ and the more probable order of markers within each linkage group was determined. The addition of 38 loci showing distorted segregation ratios (including apospory) showed 1 of them unlinked, 8 scattered in 9 different linkage groups, 25 mapping together in a single co-segregation group (M17a) and 4 associated with 2 previously unlinked SDAF constituting another group (M17b). Finally, 2 out of the 8 SDAFs added at LOD $=2.0$, grouped defining a putative new co-segregation group (M14b). The Q4117 map was built of 183 loci distributed in 39 co-segregation groups over $2,265.7 \mathrm{cM}$ with an average distance between markers of $12.4 \mathrm{cM}$. On average, each linkage group was defined by 4.6 markers spanning approximately $58 \mathrm{cM}$ (Fig. 3). Analysis of markers linked in repulsion-phase allowed the association of 25 co-segregation groups in 12 putative homologous chromosomes of 2 or 3 groups. Three previously unlinked SDAFs showed linkage in repulsion-phase with markers corresponding to groups M9a, M15a and M19a and were incorporated to the map (Fig. 3). Distances of markers linked in repulsion-phase were higher than $33.3 \mathrm{cM}$, except for the linkage groups M17a (which includes the apospory locus and several markers linked to it) and M17b, which showed $\mathrm{R}$ values around $17 \mathrm{cM}$ as well as 2 RFLP markers of groups M14a and M14b. However, the overall ratio between the numbers of pairs of undistorted SDAFs linked in repulsion vs. coupling phase (23/208) corresponded to the expected values 0-0.25:1 typical for tetrasomic inheritance (Qu and Hancok 2001). The expected genome length at $\mathrm{LOD}=2.0$ was calculated as $2,708 \mathrm{cM}$, indicating that the recombination map

Fig. 3 Linkage map of aposporous tetraploid $(2 \mathrm{n}=$ $4 \mathrm{x}=40) P$. notatum genotype Q4117. Markers' names and distances in $\mathrm{cM}$ (Kosambi) are indicated on the right and left respectively. SDAF co-segregation groups were mapped at LOD values 4.0-3.0. BSDF markers (underlined) were allocated at $\mathrm{LOD}=3.0$. Asterisks indicate markers showing distorted segregation ratios. Italics indicate markers added at $\mathrm{LOD}=2.0$. Where possible, homologous groups were aligned on the basis of SDAF linked in repulsion-phase (horizontal lines). Letters indicated homologous chromosomes 

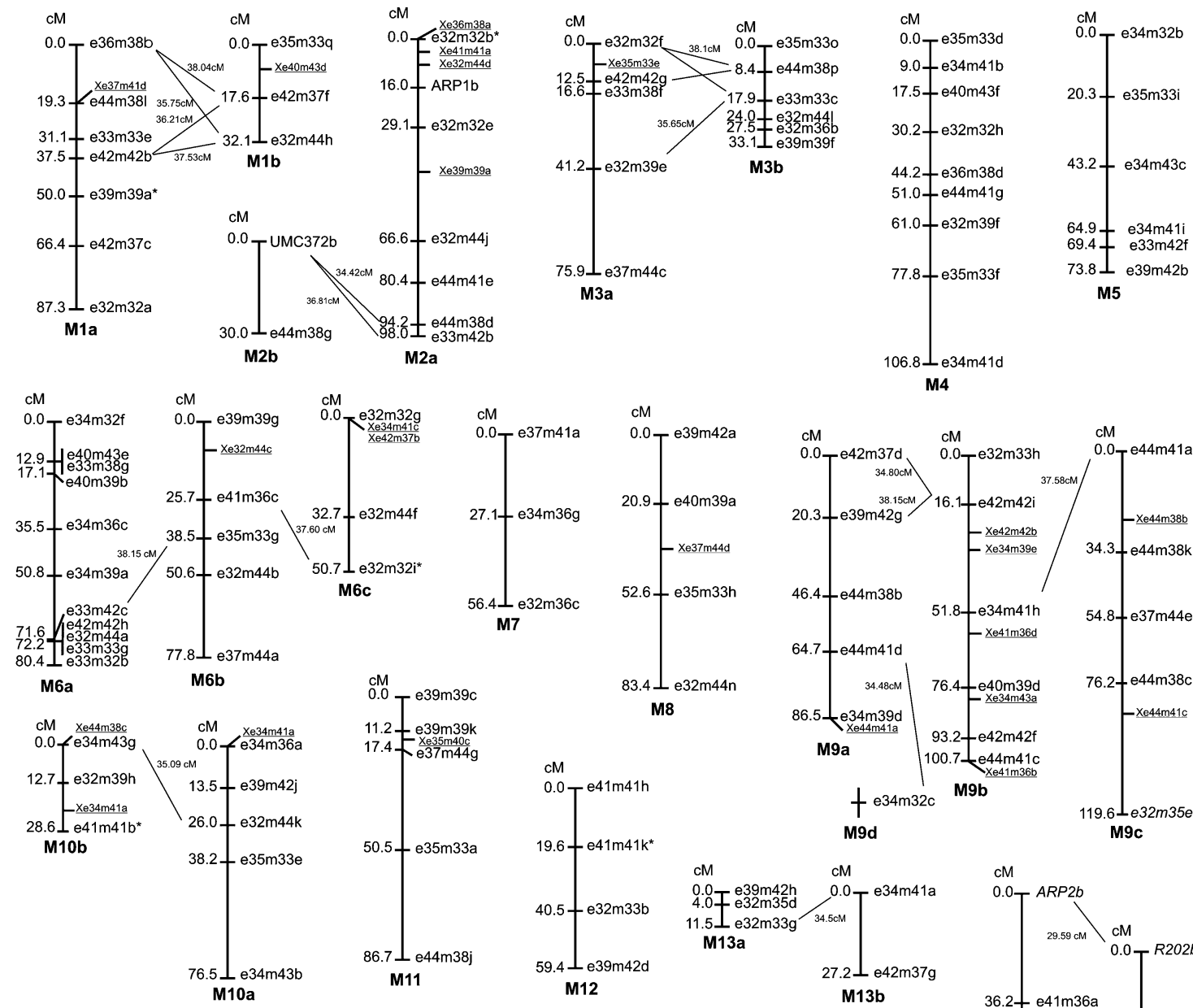
covers about $83 \%[(2,265.7 \mathrm{cM} / 2,708 \mathrm{cM}) \times 100]$ of the total genome size. Thirty three BSDFs were incorporated into 24 co-segregation groups. Linkage of three BSDFs to markers mapping in two different co-segregation groups (Xe34m41a to markers in M10a and M10b; Xe41m41b to markers in M18a and M18b; and Xe42m42a to markers in M19a and M19b) confirmed the association of homologous groups previously detected by repulsion-phase analysis (Fig. 3).

The linkage group carrying apospory was characterized by 25 loci linked in coupling phase over a $33.8 \mathrm{cM}$. The main features of the group are in agreement with a previous work focused on this specific genomic area carried out by Stein et al. 2004. Briefly, all markers within the group showed distorted segregation ratios $\left(28.7 \leq \chi^{2} \leq 68.8\right)$. A group of them defined a linkage block (apo-block) where 15 loci appeared completely linked to apospory and 7 others were located on each side with recombination values ranging between 0.7 and $4.6 \mathrm{cM}$ (Fig. 4). Analysis of linkages in repulsion-phase showed that the apo-block associated with 3 markers (e $37 \mathrm{~m} 41 \mathrm{~b}$,
Fig. 4 Male (M) and female (F) homologous chromosomes carrying the control for apospory in $P$. notatum and the putative four male homologous set. Markers names and distances in cM (Kosambi) are indicated on the right and the left, respectively.

SDAF and BSDF (underlined) were mapped at LOD values 4.0-3.0. Female and male homologous chromosomes were identified by BSDF mapping in both groups (joined by vertical lines). Asterisks indicate markers showing distorted segregation ratios. Brackets specify markers for which no unique place was found

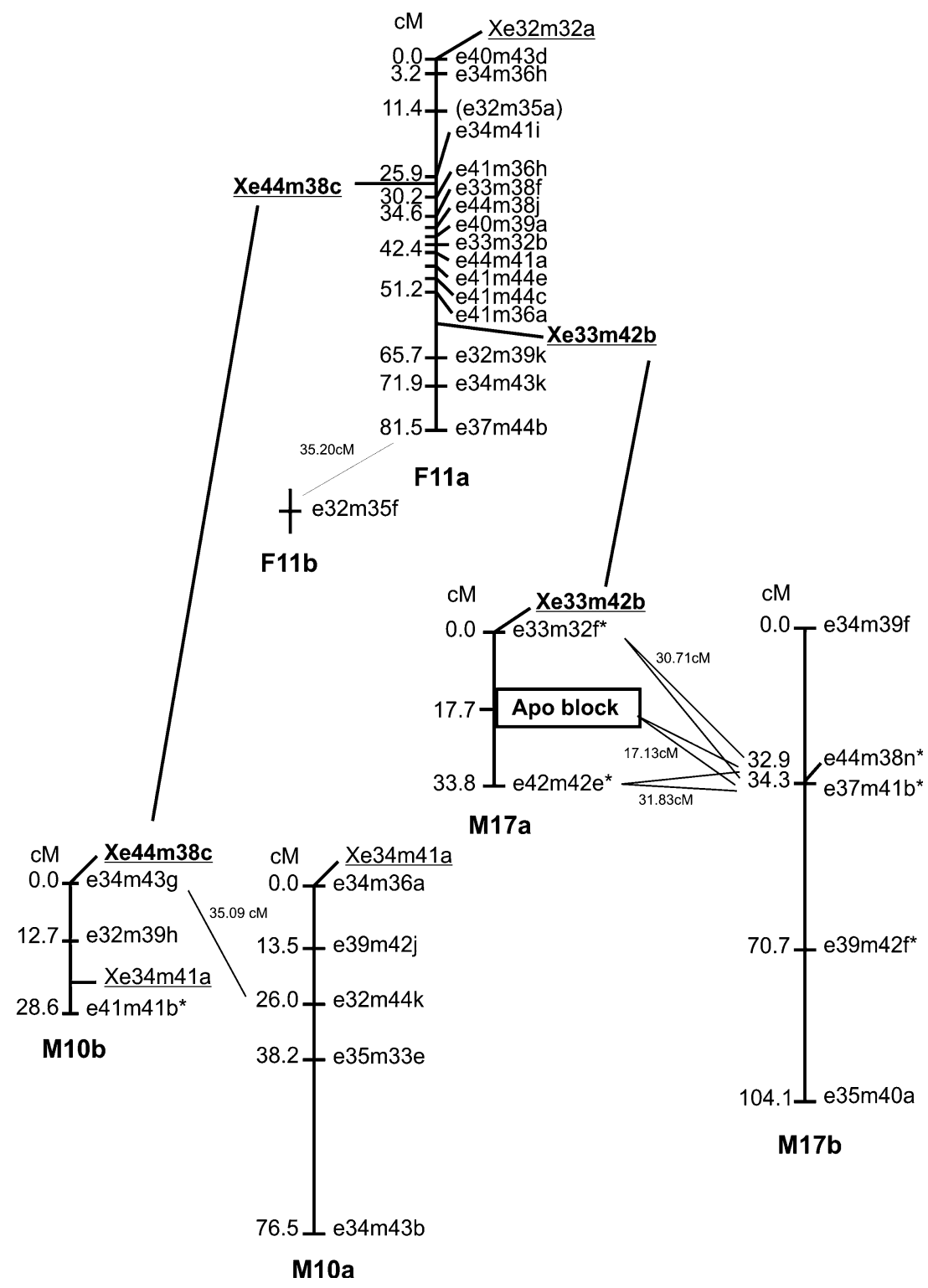


e $44 \mathrm{~m} 38 \mathrm{q}$ and $\mathrm{e} 44 \mathrm{~m} 38 \mathrm{n}$ ) of one homologous chromosome, with $\mathrm{R}$ values varying among 15.7 and $21 \mathrm{cM}$, all of them lower than the expected $\mathrm{Ri}$ $(33.3 \mathrm{cM})$ for a true autotetraploid. These values confirmed the preferential chromosome pairing in this particular genomic region reported previously (Stein et al. 2004). The addition of BSDF allowed the identification of the putative complete set of homologs for the chromosome carrying the apospory locus (see below).

\section{Mapping RFLP makers}

Thirty nine heterologous clones (29 from rice and 10 from maize) distributed over the rice and maize genetic maps were tested on $P$. notatum DNA. Only 9 RFLP clones (23.0\%) (RZ273, RZ567, RZ630, R202, C1069, R642, R3375, $U M C 372, U M C 132)$ displayed polymorphisms between parents. The rest produced a monomorphic hybridization pattern $(30.8 \%)$ or did not show clear hybridization signals because of faint bands or smearing (46.2\%). Clones RZ630, R642 and UMC132 could not be mapped because polymorphic fragments were not segregating in the population. The remaining 6 clones produced 8 markers ( 3 female and 5 male) that were located as follows: RZ273 on F5a; UMC372 on F5a and M2b; RZ567 on F17 and M21; R202 on M14b; C1069 on M17a and R3375 on M14b (Figs. 2, 3). Hybridizations performed with homologous clones ARP1 and ARP2 (both related to the aposporous embryo sac development, Pessino et al. 2001 and Pessino SC personal communication), generated markers that mapped on linkage groups $\mathrm{F} 3 \mathrm{c} \quad(A R P 1 a), \quad \mathrm{M} 2 \mathrm{a} \quad(A R P 1 b), \quad \mathrm{F} 9 \mathrm{a}$ $(A R P 2 a)$ and M14a (ARP2b). cDNA clone $S R P 1$ (associated to sexual embryo sac development (Pessino SC personal communication) did not show polymorphism between parents and could not be mapped. On the other hand, only one clone from Brachiaria sp of the total 7 tested (clone 18) could be mapped successfully, and it was located on linkage group F6a.

Identification of homologous chromosomes of Q4188 and Q4117

Twenty one biparental single dose markers (BSDF) mapped in linkage groups of both Q4118 and Q4117. They were used for detecting 10 female and male homologous chromosomes (Table 3). One of these associations was confirmed by hybridization of cDNA $A R P 1$, which generated markers on groups $\mathrm{F} 3 \mathrm{c}$ and $\mathrm{M} 2 \mathrm{a}$. Interestingly, $\mathrm{X} 33 \mathrm{~m} 42 \mathrm{~b}$ mapped into the group carrying the locus controlling apospory (M17b) and F11a. This female group is characterized by 19 markers over $81.5 \mathrm{cM}$, with a cluster of 11 markers concentrated on $25 \mathrm{cM}$. Putative allelic AFLP fragments generated by 5 primer combinations (e33m32, e41m44, e32m35, e40m43, e44m38) mapped to both groups, thus supporting the chromosome association. In addition, Xe44m38c present in F11a, mapped to male group M10b. This relationship indicated that M17a, M17b, M10a and M10b would be the four homologous chromosomes of the set bearing the factor/s responsible for apospory. Because we were not able to detect a direct association

Table 3 Female and male homologous chromosomes identified by BSDFs

\begin{tabular}{lll}
\hline BSDF & Homologous female and male groups \\
\cline { 2 - 3 } & Q4188 & Q4117 \\
\hline Xe41m41a, Xe32m44d, Xe36m38a, Xe39m39a & F3 (a/b/c) & M2 (a/b) \\
Xe35m40c, Xe32m36b, Xe42m42a & F5 (a/b/c) & M11 + M19 (a/b) \\
Xe40m43b, Xe41m41b & F6 (a/b) & M18 (a/b)+ M22 \\
Xe41m36b, Xe44m41a, Xe44m41c & F8 + F18 & M9 (a/b/c/d) \\
Xe35m33e & F9 (a/b) & M3 (a/b) \\
Xe40m39f & F10 & M14 (a/b) \\
Xe33m42b, Xe44m38c & F11(a/b) & M17 (a/b) + M10 (a/b) \\
Xe37m41c & F12 & M16 \\
Xe32m44c, Xe42m37b, Xe34m41c & F13 + F15 (a/b) & M6 (a/bc) \\
Xe32m35a & F16 & M20 \\
\hline
\end{tabular}


between M10 and M17 so far, this hypothesis still should be tested (Fig. 4).

\section{Discussion}

We report here the first genetic linkage map of tetraploid Paspalum notatum Flügge based on molecular markers. The construction of the present map allowed the identification of the linkage groups associated with apomixis in the species as well as a general characterization of its mode of inheritance and genome organization.

Six hundred and eighty seven molecular markers were generated for developing two maps (maternal and paternal) with the segregation information derived from each parental genotype. Most markers showed a 1:1 segregation ratio indicating its heterozygous simplex state, either in Q4188 or Q4117 plants. This condition facilitated the identification of SDAFs for mapping at the polyploid level and the achievement of acceptable genome coverage. About $9 \%$ of the markers corresponded to BSDF (3:1), which were informative for identifying homologous groups between maternal and paternal maps (Table 3). Approximately, 5\% and $13 \%$ of markers segregating from the female and male parents respectively, showed distorted segregation ratios. Most of the distorted markers originated from Q4117 (like the apospory locus) were characterized by being under-represented in the progeny (Fig. 1). Segregation distortion could be caused by partial lethal factors, i.e. elimination of gametes or zygotes controlled by a partial lethal factor located in the region neighbouring the marker (Cheng et al. 1998). The presence of a partial lethal factor was proposed for explaining the segregation distortion of apospory in $P$. notatum by Martínez et al. (2001). Similar factor/s could be acting at other loci across the genome and being responsible for the segregation distortion observed in some AFLP markers. Interestingly, the distribution of distorted markers differed among female and male maps. In the former most of them appeared scattered in several linkage groups, while in Q4117 the majority (24/ 38) mapped together with apospory in a single linkage group (M17a). This is a strong indication that this particular region is actually affected by some partial lethal effect as proposed by Martínez et al. 2001.

A total of 263 and 216 markers were mapped on Q4188 and Q4117 genomes. Lengths of the genetic maps for the female and male parents were estimated to be $1,815 \mathrm{cM}$ and $2,708 \mathrm{cM}$ respectively, indicating an acceptable genomic coverage by the recombination maps $(83 \%$ and $87 \%$ ). A higher recombination frequency in male gametes was observed. Variation in length of parental maps was reported in several species like ryegrass (Warnke et al. 2004), Festuca arundinacea (Saha et al. 2005), Fragaria $x$ ananassa (Lecrceteua-Köheler et al. 2003) and bermudagrass (Bethel et al. 2006) and it could be reflecting a higher recombination frequency in the male gametes.

Several groups were densely populated with markers (i.e. groups F2, F3c, F5a and M17a), but others were only sparsely mapped. Moreover, 74 (42 female and 32 male) SDAF remained unlinked and several repulsion-phase linkages involved single unlinked markers in both maps. These results are clear indications that although relatively high genome coverage was achieved, the map is still incomplete. Presumably, as map density increases, unlinked markers will be added to the existent or to new linkage groups allowing the identification of the complete chromosome sets. Since most markers linked in repulsion phase resulted in linkage distances $(\mathrm{R})$ greater than $33.3 \mathrm{cM}$, our previous conclusion about a general tetrasomic inheritance for the species was confirmed (Stein et al. 2004). This condition should be considered for developing appropriate breeding strategies aiming the incorporation of agronomic interest traits into cultivated strains.

The efficiency for detecting co-segregation groups differed between the female and male maps (26 vs. 39). One of the explanations for this outcome could be the origin of both parental genotypes. Q4188 was obtained experimentally from a cross between Q3664 (used as female parent) and Q3853 (used as pollen donor) (Quarin et al. 2003). Q3664 is a tetraploid facultative apomictic plant with high levels of sexuality, derived from a sexual tetraploid (PT-2) obtained 
by colchicine treatment of a diploid ( $P$. notatum var. saurae) (Quarin et al. 2003)]. This recentlyinduced polyploid constitution could have given rise to many loci at a multiplex condition (i.e. no segregation), reducing the efficiency for detecting linkages in some genomic areas. On the other hand, Q4117 is a natural genotype whose actual genetic constitution could have derived from evolution through many generations and it is possible that many loci are in a simplex heterozygous state, offering more possibilities for detecting linkages in both coupling and repulsion phases.

The DNA content of diploid $P$. notatum var. saurae was estimated on $0.57 \mathrm{pg} / \mathrm{C}$ (or $554 \mathrm{Mbp}$ ) (Jarret et al. 1995). Consequently, the gametes of the autotetraploids would carry some $1.14 \mathrm{pg}$ (1,108 Mbp). Considering the total length of the recombination maps of Q4188 and Q4117, $1 \mathrm{cM}$ would be approximately $696 \mathrm{kpb}(1,108 \mathrm{Mbp} /$ 1,590.6 cM) and $489 \mathrm{kpb}(1,108 \mathrm{Mbp} / 2,265.7 \mathrm{cM})$, respectively. These values are within the range expected for plants and comparable to those estimated from the diploid map of the species, in which $1 \mathrm{cM}$ would equal $559 \mathrm{kpb}$ (554 Mbp/ $991 \mathrm{cM})$ (Ortiz et al. 2001).

Since AFLP maps cannot be used directly for comparative mapping, we tested a group of RFLP probes distributed over the rice and maize maps as starting point for future investigations on this topic. However, since only 6 probes could be mapped and partially tie this map to others, we were unable to make any rational comparative analysis. A more extensive screening and mapping of anchor markers will be necessary for addressing this point. On the other hand, rice clone C1069 mapped completely linked to apospory as expected (Martínez et al. 2003, Stein et al. 2004), but none of the P. notatum or B. brizhantha cDNA clones (ARP1, ARP2, SPR1 and 18), previously associated with the development of aposporous and non-aposporous embryo sacs generated fragments positionally associated with apospory. These results indicated that these clones hold only a functional (rather than a genetic) connection with the aposporous/sexual phenotypes and may correspond to genes involved in the cascade of events leading to embryo sac formation.
The linkage group carrying the locus controlling apospory (M17a) was identified in Q4117. It showed particular characteristics that differentiated it from the rest of the groups detected in the whole map, which are in agreement with previous results obtained in the species (Martínez et al. 2003; Stein et al. 2004). Although it is difficult to make an estimation of the physical length of the region involved (due to the segregation distortion and restriction in recombination) the apo-block detected could be a large chromosome fragment. This characteristic could seriously complicate the map based cloning strategies for isolating the genes responsible for apospory. Consequently, a comparative approach or reverse genetic methods should be considered for achieving this goal. Similarly, a large structure was found associated to apospory in Pennisetum squamulatum and Cenchrus ciliaris (Akiyama et al. 2005). Interestingly, one BSDF marker (Xe33m42b) that mapped in group M17a allowed the identification of the putative female homologue and by extension the putative complete set of homologues (Fig. 3). This genome region justifies further investigation because allelic forms of the apospory/sexuality determinant/s could be included within it.

The map presented here can be used as a genetic framework for basic and applied studies in Paspalum and represents the foundation for a denser map. It establishes the first example of a genetic linkage map of tetraploid $P$. notatum with both sexual and apomictic forms. Future identification of markers linked to complex traits such us cold resistance, digestibility or dry matter production, could be exploited for marker assisted selection-breeding programs aimed at the improvement of the existent cultivars of the species, many of which are important forage crops.

Acknowledgements Authors wish to thank to Glaucia Cabral and Dr. Vera Tavares de Campos Carneiro, EMBRAPA Cenargen-Brazil for providing the cDNA probes from Brachiaria $s p$. We thank Ing. Florencia Galdeano for her technical assistance. This study was financed by the Agencia Nacional de Promoción Científica y Tecnológica (ANPCyT), Argentina, PICT $\mathrm{N}^{\circ} 13578$ and PAV 137/3. Consejo Nacional de Investigaciones Científicas y Técnicas (CONICET), Argentina, PIP No. 6805. Centro Argentino Brasilero de Biotecnología 
(CABBIO 2003 No. 012). J. Stein and MP Rodriguez received fellowships from CONICET and ANPCyT, respectively. EJ Martínez, SC Pessino, CL Quarin and JPA Ortiz are career members of CONICET.

\section{References}

Akiyama Y, Hanna WW, Ozias-Akins P (2005) High resolution physical mapping reveals that the apospory specific genomic region (ASGR) in Cenchrus ciliaris is located on a heterochromatic and hemizygous region of a single chromosome. Theor Appl Genet 111:10421051

Al-Janabi SM, Honeycutt RJ, McClelland M, Sobral BW (1993) A genetic linkage map of Saccharum spontaneum L. SES 208. Genetics 134:1249-1260

Bethel CM, Sciara EB, Estill JC, Bowers JE, Hanna W, Paterson AH (2006) A framework map of bermudagrass (Cynodon dactylon $\mathrm{x}$ transvaalensis) based on single-dose restriction fragments. Theor Appl Genet 112:727-737

Burton GW (1948) The method of reproduction of common bahiagrass, Paspalum notatum. J Am Soc Agron 40:443-452

Burton GW (1967) A search for the origin of Pensacola bahiagrass. Econ Bot 21:379-382

Chakravarti A, Lasher LK, Reefer JE (1991) A maximum likelihood method for estimating genome length using genetic linkage data. Genetics 128:175-182

Chase A (1929) North American species of Paspalum. Cont US Natl Herb (28): Part 1

Cheng R, Kleinhofs A, Ukai Y (1998) Method for mapping a partial lethal-factor locus on a molecularmarker linkage map of a backcross and doubledhaploid population. Theor Appl Genet 97:293-298

Da Silva JAG, Sorrells ME, Burnquist WL, Tanskley SD (1993) RFLP linkage map and genome analysis of Saccharum spontaneum. Genome 36:782-791

Daurelio DL, Espinoza F, Quarin CL, Pessino SC (2004) Genetic diversity in sexual diploid and apomictic tetraploid populations of Paspalum notatum situated in sympatry or allopatry. Plant Syst Evol 244:189-199

Ebina M, Nakagawa H, Yamamoto T, Araya H, Tsuruta S, Takahra M, Nakayima K (2005) Co-segregation of AFLP and RAPD markers to apospory in Guineagrass (Panicum maximum Jacq.). Grassland Sci 51:7178

Espinoza F, Daurelio DL, Pessino SC, Valle EM, Quarin CL (2006) Genetic characterization of Paspalum notatum accessions by AFLP markers. Plant Syst Evol 258:147-159

Forbes I, Burton GW (1961) Cytology of diploids, natural and induced tetraploids, and intraspecific hybrids of bahiagrass, Paspalum notatum. Flugge. Crop Sci 1:402-406

Gates RN, Quarin CL, Pedreira CGS (2004) Bahiagrass. In: Moser LE, Burson BL, Sollenberger LE (eds) Warm-season (C4) grasses. ASA, CSSA, and SSSA, Madison, WI pp 651-680
Grattapaglia D, Sederoff R (1994) Genetic linkage maps of Eucalyptus grandis and Eucalyptus urophylla using a pseudo test cross: mapping strategy and RAPD markers. Genetics 137:1121-1137

Jarret RL, Ozias-Akins P, Phatak S, Nadimpalli R, Duncan R, Hiliard S (1995) DNA contents in Paspalum ssp. determined by flow cytometry. Genet Resour Crop Evol 42:273-242

Jessup RW, Burson BL, Burrow G, Wang YW, Chang C, Li Z, Paterson AH, Hussey MA (2003) Segmental allotetraploidy and allelic interactions in buffelgrass (Pennisetum ciliare (L.) Link syn. Cenchrus ciliaris L) as revealed by genome mapping. Genome 46:304-313

Kosambi DD (1944) The estimation of map distance from recombination values. Ann Eugen 12:172-175

Lander ES, Green P, Abrahamson J, Barlow A, Daly MJ, Lincoln SE, Newburg L (1987) MAPMAKER: an interactive computer package for constructing primary genetic linkage maps of experimental and natural populations. Genomics 1:174-181

Lerceteau-Kölher E, Guerin G, Laigret F, DenoyesRothan B (2003) Characterization of mixed disomic and polysomic inheritance in octoploid strawberry (Fragaria $x$ ananassa) using AFLP mapping. Theor Appl Genet 107(4):619-628

Maliepaard C, Alston FH, van Arkel G, Brown LM, Chevreau E, Dunemann F, Evans KM, Gardiner S, Guilford P, van Heusden AW, Janse J Laurens F, Lynn JR, Manganaris AG, den Nijs APM, Periam N, Rikkerink E, Roche P, Ryder C, Sansavini S, Schimidt H, Tartarini S, Verhaegh JJ, Vrielink-van GM, King GJ (1998) Aligning male and female linkage maps of apple (Malus pumila Mill.) using multi-allelic markers. Theor Appl Genet 97:60-73

Martínez EJ, Urbani MH, Quarin CL, Ortiz JPA (2001) Inheritance of apospory in bahiagrass, Paspalum notatum. Hereditas 135:19-25

Martínez EJ, Hopp E, Stein J, Ortiz JPA, Quarin CL (2003) Genetic characterization of apospory in tetraploid Paspalum notatum based on the identification of linked molecular markers. Mol Breed 12(4):319-327

Missaoui AM, Paterson AH, Bouton JH (2005) Investigation of genomic organization in switchgrass (Panicum virgatum L.) using DNA markers. Theor Appl Genet 110:1372-1383

Nogler GA (1984) Gametophytic apomixis. In: Johri BM (ed) Embryology of Angiosperms. Springer-Verlag, Berlin

Ortiz JPA, Pessino SC, Bhat V, Hayward MD, Quarin CL (2001) A genetic linkage map of diploid Paspalum notatum. Crop Sci 41:823-830

Pessino SC, Evans C, Ortiz JPA, Armstead I, do Valle CB, Hayward MD (1998) A genetic map of the aposporyregion in Brachiaria hybrids: identification of two markers closely associated with the trait. Herditas 128:153-158

Pessino SC, Espinoza F, Martínez EJ, Ortiz JPA, Valle E, Quarin CL (2001) Isolation of cDNA clones differentially expressed in flowers of apomictic and sexual Paspalum notatum. Hereditas 134:35-42 
Porceddu A, Albertini E, Barcaccia E, Falistocco E, Falcinelli M (2002) Linkage mapping in apomictic and sexual Kentucky bluegrass (Poa pratensis L.) genotypes using a two way pseudo-testcross strategy based on AFLP and SAMPL markers. Theor Appl Genet 104:273-280

Pupilli F, Martinez EJ, Busti A, Calderini O, Quarin CL, Arcioni S (2004) Comparative mapping reveals partial conservation of synteny at the apomixis locus in Paspalum spp. Mol Gen Genomics 270:539-548

Qu L, Hancock JF (2001) Detecting and mapping repulsion-phase linkage in polyploids with polysomic inheritance. Theor Appl Genet 103:136-143

Quarin CL, Burson BL, Burton GW (1984) Cytology of intra and interspecific hybrids between two cytotypes of Paspalum notatum and P. cromyorrhyzon. Bot Gaz 145:420-426

Quarin CL, Urbani MH, Blount AR, Martínez EJ, Hack CM, Burton GW, Quesenberry KH (2003) Registration of Q4188 and Q4205, sexual tetraploid germplasm lines of Bahiagrass. Crop Sci 43:745-746

Ripol MI, Churchill GA, Da Silva JAG, Sorrells M (1999) Statistical aspects of genetic mapping in autopolyploids. Gene 235:31-41

Ritter E, Gebhardt C, Salamini F (1990) Estimation of recombination frequencies and construction of RFLP linkage maps in plants from crosses between heterozygous parents. Genetics 125:645-654
Rodrigues JCM, Cabral GB, Dusi DMA, de Mello LV, Ridgen DJ, Carneiro VTC (2003) Identification of differentially expressed cDNA sequences in ovaries of sexual and apomictic plants of Brachiaria brizantha. Plant Mol Biol 53:745-757

Saha MC, Mian R, Zwonitzer JC, Chekhovskiy K, Hopkins AA (2005) An SSR and AFLP based genetic linkage map of tall fescue (Festuca arundinacea Schreb.). Theor Appl Genet 110(2):323-336

Stam P (1993) Construction of integrated genetic linkage maps by means of a new computer package: JoinMap. Plant J 3:739-744

Stein J, Quarin CL, Martínez EJ, Pessino SC, Ortiz JPA (2004) Tetraploid races of Paspalum notatum show polysomic inheritance and preferential chromosome pairing around the apospory-controlling locus. Theor Appl Genet 109:186-191

Warnke SE, Barker RE, Jung G, Sim S-C, Mian MAR, Saha MC, Brilman LA, Dupal MP, Foster JW (2004) Genetic linkage mapping of an annual x perennial ryegrass population. Theor Appl Genet 109:294-304

Wu KK, Burnquist W, Sorrells ME, Tew TL, Moore PH, Tanksley SD (1992) The detection and estimation of linkage in polyploids using single-dose restriction fragments. Theor Appl Genet 83:294-300 